\title{
L'IMAGINAIRE ET LA RELIANCE DANS LA SOCIOLOGIE DE DURKHEIM
}

Jean-Martin Rabot

De Boeck Supérieur | «Sociétés »

2015/1 n 127 | pages 25 à 40

ISSN 0765-3697

ISBN 9782807301320

Article disponible en ligne à l'adresse :

http://www.cairn.info/revue-societes-2015-1-page-25.htm

\section{!Pour citer cet article :}

Jean-Martin Rabot, «L'imaginaire et la reliance dans la sociologie de Durkheim », Sociétés 2015/1 (n 127), p. 25-40.

DOI $10.3917 /$ soc. 127.0025

Distribution électronique Cairn.info pour De Boeck Supérieur.

(C) De Boeck Supérieur. Tous droits réservés pour tous pays.

La reproduction ou représentation de cet article, notamment par photocopie, n'est autorisée que dans les limites des conditions générales d'utilisation du site ou, le cas échéant, des conditions générales de la licence souscrite par votre établissement. Toute autre reproduction ou représentation, en tout ou partie, sous quelque forme et de quelque manière que ce soit, est interdite sauf accord préalable et écrit de l'éditeur, en dehors des cas prévus par la législation en vigueur en France. Il est précisé que son stockage dans une base de données est également interdit. 


\section{L'IMAGINAIRE ET LA RELIANCE DANS LA SOCIOLOGIE DE DURKHEIM}

Jean-Martin RABOT*

\section{La signification du positivisme durkheimien}

Le positivisme durkheimien n'a jamais fait l'ombre d'un doute. Dans Les règles de la méthode sociologique, Durkheim s'est fixé la tâche de définir a priori le statut de la sociologie en se réclamant explicitement du rationalisme, entendu comme modèle théorique et pratique : "Notre principal objectif, en effet, est d'étendre à la conduite humaine le rationalisme scientifique, en faisant voir que, considérée dans le passé, elle est réductible à des rapports de cause à effet qu'une opération non moins rationnelle peut transformer ensuite en règles d'action pour l'avenir ${ }^{1}$. " La science sociologique a donc pour vocation de faire accéder ce qui est encore irrationnel à l'ordre du rationnel en le rendant intelligible et d'apporter ainsi sa contribution à un projet de transformation de la société selon les canons de la raison.

La méthode de Durkheim est orientée, sinon vers une amélioration de la société, du moins vers une résolution des maux qui la touchent. Il suffit pour cela que la sociologie parvienne "à déterminer les lois générales de la société, comparables aux lois de la nature, qui détermineront une sorte de technique sociale comme il existe une technique matérielle ${ }^{2}$. Comme le sous-entend encore J. Freund, c'est la notion de "nature des choses » qui constitue le fondement de sa croyance en la possibilité d'améliorer les processus de socialisation. S'il existe une nature des choses pour tout ce qui touche au monde humain, c'est qu'il est possible de

* Professeur à l'Institut des Sciences Sociales de l'Université du Minho et membre du CECS (Centro de Estudos de Comunicação e Sociedade), Braga, Portugal.

1. É. Durkheim, Les règles de la méthode sociologique, PUF, Paris, 1973a, p. IX.

2. J. Freund, Philosophie et sociologie, Cabay, Louvain-la-Neuve, 1984, p. 279. 
soumettre les faits sociaux à des lois. Durkheim se réfère d'ailleurs à une citation de Montesquieu où les deux termes sont explicitement mis en rapport : " Les lois sont les rapports nécessaires qui dérivent de la nature des choses ${ }^{3}$. "Durkheim répète l'idée de Montesquieu en légitimant le caractère scientifique de la sociologie dans un texte publié en 1909 et repris dans La science sociale et l'action : "La sociologie ne pouvait apparaître avant qu'on eût acquis le sentiment que les sociétés, comme le reste du monde, sont soumises à des lois qui dérivent nécessairement de leur nature et qui l'expriment ${ }^{4}$. »

Lenjeu de ces définitions réside dans la possibilité de l'affirmation de l'objectivité intrinsèque de la réalité sociale. La société constitue ainsi un substrat rationnel qu'il suffit de rendre intelligible au moyen de l'édification de lois scientifiques pour la guider vers un avenir plus radieux. Le fait même que les faits sociaux puissent être considérés comme des choses constitue l'un des gages les plus sûrs pour l'établissement de lois. Il y a donc antécédence et suprématie des choses par rapport à la volonté en ceci qu'elles la déterminent. L'un des interprètes de Durkheim a précisément montré que le thème obsédant de l'intégration sociale chez Durkheim ne répond pas tant à une forme de conservatisme héritée de la tradition philosophique qu'à une interrogation de fond : "Comment la société tient-elle et se transforme-t-elle si le législateur n'en est pas le souverain maître 5 ? » Si l'individu est mû par des causes objectives qui dérivent de la nature des choses, la question de la surdétermination du réel par l'apport d'une causalité humaine ne se pose plus.

Cette relativisation des facultés d'autonomie et des possibilités d'action de l'homme nous montre bien que le positivisme de Durkheim n'est pas à prendre au pied de la lettre. Le naturalisme sociologique de Durkheim n'implique pas que la sociologie soit réduite à une physique sociale. Il prend d'ailleurs soin de distinguer le caractère spécifique de son naturalisme dans la définition qu'il propose des faits sociaux, lorsqu'il affirme que ceux-ci ne sont pas à proprement parler des choses matérielles, mais "des choses au même titre que les choses matérielles " ${ }^{6}$. Le sociologue n'est donc pas un physicien, même s'il adopte la même démarche que lui par rapport à son propre objet d'étude. Comme toute science, la sociologie se doit de définir son objet. En tant que science des faits sociaux, elle a à chercher quels sont les faits qui peuvent être qualifiés de sociaux et quels sont les caractères qui les distinguent spécifiquement des autres faits.

Ce qui définit le fait social, c'est justement qu'il est extérieur à l'individu et qu'il le transcende. S'imposant à lui, l'individu n'a d'autre choix que de s'y conformer librement. La contrainte sociale, en tant que faisant partie de la nature des choses, est ce qui caractérise par excellence le fait social, puisqu'elle « ne dérive pas d'un

3. Montesquieu, cité par É. Durkheim, Montesquieu et Rousseau précurseurs de la sociologie, Marcel Rivière, Paris, 1966, p. 75.

4. É. Durkheim, La science sociale et l'action, PUF, Paris, 1970, p. 138.

5. B. Lacroix, Durkheim et le politique, Presses de la fondation nationale des sciences politiques (Université de Montréal), Montréal, 1981, pp. 305-306.

6. É. Durkheim, Les règles de la méthode sociologique, PUF, Paris, 1973a, p. XII. 
arrangement conventionnel que la volonté humaine a surajouté de toutes pièces au réel ; elle sort des entrailles même de la réalité ; elle est le produit nécessaire de causes données. Aussi, pour amener l'individu à s'y soumettre de son plein gré, n'est-il nécessaire de recourir à aucun artifice : il suffit de lui faire prendre conscience de son état de dépendance et d'infériorité naturelles - qu'il s'en fasse par la religion une représentation sensible et symbolique ou qu'il arrive à s'en former par la science une notion adéquate et définie ${ }^{7}$. " Loin d'être réactionnaire, la méthode de Durkheim qui consiste à inscrire les faits sociaux dans la nature des choses constitue une critique des facultés d'autonomie que l'on attribue avec trop de largesse à la raison humaine. En d'autres termes, il n'est point possible pour l'homme de faire table rase du passé pour tout créer ou recréer.

C'est dans un souci de minimisation du principe de l'autonomie que Durkheim a analysé la fonction de l'éducation dans la vie sociale. Il part du postulat que le fait que l'éducation ait varié au cours du temps, étant d'essence individualiste chez les Romains, chrétienne au Moyen Âge, laïque à partir de la Renaissance, et de caractère scientifique de nos jours, n'invalide nullement que les hommes ne se méprennent pas sur ce qu'elle doit être. De ce point de vue, la pluralité des systèmes culturels est un gage de l'homogénéité du tout. Au-delà des différences culturelles, il existe un fond commun garantissant la sociabilité : "Il n'y a pas de peuples où il n'existe un certain nombre d'idées, de sentiments et de pratiques que l'éducation doit inculquer à tous les enfants indistinctement, à quelque catégorie sociale qu'ils appartiennent. [...] Si chaque caste, chaque famille a ses dieux spéciaux, il y a des divinités générales qui sont reconnues de tout le monde et que tous les enfants apprennent à adorer ${ }^{8}$. „ Durkheim revient d'innombrables fois sur l'idée du rôle moralisateur de l'enseignement : "Détacher les esprits des vues égoïstes et des intérêts matériels; remplacer la piété religieuse qui s'en va par une sorte de piété sociale. Or ce n'est ni avec la règle de trois, ni avec le principe d'Archimède qu'on pourra jamais moraliser les foules. Il n'y a que la culture esthétique qui puisse aussi profondément agir sur les âmes ${ }^{9}$. " L'amour d'autrui n'est donc qu'une transfiguration de l'amour du beau. Et c'est au système éducatif qu'il incombe de les enseigner et de les cultiver.

La transmission aux individus de sentiments communs et l'apprentissage initiatique d'un partage des émotions par le biais de l'éducation constituent justement l'une des façons par laquelle la société manifeste son caractère transcendant. En d'autres termes, la transcendance est immanente à la société. Sachant que l'homme ne peut vivre en dehors de la société, à l'écart des normes qui font d'elle ce qu'elle est, il n'a d'autre solution que de la juger bonne et même d'arriver à l'aimer. Durkheim nous montre que si les formes et les contenus de l'éducation peuvent changer, par exemple " endurcir les corps à la fatigue » comme à Sparte, "faire des corps beaux à la vue » comme à Athènes, "former des guerriers agiles

7. Ibid., pp. 121-122.

8. É. Durkheim, Éducation et sociologie, PUF, Paris, 1985, p. 49.

9. É. Durkheim, La science sociale et l'action, PUF, Paris, 1970, p. 176. 
et souples " comme au temps de la chevalerie, ou encore se faire dans " un but hygiénique » afin de " contenir les dangereux effets d'une culture intellectuelle trop intense ${ }^{10}$, elle répond cependant à un même besoin qui est d'immerger notre être individuel dans l'être collectif, entendu comme « système d'idées, de sentiments, d'habitudes qui expriment en nous, non pas notre personnalité, mais le groupe ou les groupes différents dont nous faisons partie ${ }^{11}$. Aussi serait-il malvenu de juger l'éducation religieuse du Moyen Âge, de tendance ascétique, selon les critères scientifiques en vogue aujourd'hui, dans la mesure où " cet ascétisme était nécessaire, car la seule manière de s'adapter à la rudesse de ces temps difficiles était de l'aimer " ${ }^{12}$. L'éducation n'a d'autre but que de faire en sorte que l'homme "se sente solidaire d'une société, si humble soit-elle » ${ }^{13}$, de le relier, au sens religieux du terme, à un quelconque groupe, au moyen d'une représentation qui soit de l'ordre du vécu et du ressenti : "Il faut que cette représentation ait quelque chose d'émotif, qu'elle ait plus le caractère d'un sentiment que d'une conception ${ }^{14}$. »

Sans vouloir recourir à l'explication fétiche d'une rupture épistémologique dans l'œuvre de Durkheim, il faut bien reconnaître que la dissimilitude des principes sociologiques énoncés dans les Règles avec la pratique effective, telle qu'elle s'exprime dans les travaux postérieurs, à l'instar du Suicide, ne fait qu'annoncer l'importance croissante que Durkheim accorde aux phénomènes religieux, avec la part d'émotions qu'ils contiennent. Bien avant la publication des Formes élémentaires, Durkheim affirme dans les années 1997-1998 que les phénomènes religieux constituent « le germe d'où tous les autres - ou, tout le moins, presque tous les autres - sont dérivés. La religion contient en elle, dès le principe, mais à l'état confus, tous les éléments qui, en se dissociant, en se déterminant, en se combinant de mille manières avec eux-mêmes, ont donné naissance aux diverses manifestations de la vie collective. C'est des mythes et des légendes que sont sorties la science et la poésie ; c'est de l'ornemantique religieuse et des cérémonies du culte que sont venus les arts plastiques; le droit et la morale sont nés des pratiques rituelles. On ne peut comprendre notre représentation du monde, nos conceptions philosophiques sur l'âme, sur l'immortalité, sur la vie, si l'on ne connaît pas les croyances religieuses qui en ont été la forme première ${ }^{15}$. »

\section{Science et religion}

Il faut à tout prix éviter l'écueil selon lequel Durkheim serait "le sociologue de l'anomie ${ }^{16}$ et que toutes les études spécialisées auxquelles il s'est consacré, y

10. É. Durkheim, Éducation et sociologie, PUF, Paris, 1985, p. 55.

11. Ibid., p. 102.

12. Ibid., pp. 54-55.

13. É. Durkheim, L'Éducation morale, Fabert, Paris, 2005, p. 116.

14. Ibid., p. 285.

15. É. Durkheim, Journal sociologique, PUF, Paris, 1969, p. 138.

16. P. Besnard, L'anomie, ses usages et ses fonctions dans la discipline sociologique depuis

Durkheim, PUF, Paris, 1987, p. 127. 
compris les recherches de sociologie religieuse, seraient orientées dans le sens d'une résolution de la crise tout à fait spécifique des sociétés industrielles. Si «ce type d'interprétation est séduisant et toujours défendable " ${ }^{17}$, il ne correspond que très partiellement à la vérité. En effet, la sociologie de Durkheim ne peut être exclusivement examinée sous ce prisme. S'il attribue effectivement un caractère fonctionnel à la science sociologique, s'il cherche effectivement des solutions viables à la question de l'anomie qui taraude la société moderne, s'il suggère de faire de l'irrémédiable phénomène de l'individualisme une religion adaptée aux temps modernes, à même de suppléer à la disparition de la conscience collective due au démantèlement des structures sociales traditionnelles, comme la famille, le voisinage, le provincialisme, Durkheim étudie quand même la religion et la science en elles-mêmes, sans aucune visée utilitariste. Une lecture des rapports entre la science et la religion, indépendamment des vertus thérapeutiques qu'il prêtait à la sociologie, s'avère donc légitime.

Durkheim était pertinemment conscient du fait qu'en période d'abondance, et donc de plus grand scepticisme, comme dirait Pareto, la science ne pourrait jamais évincer les résidus religieux dont toute société est pétrie. La science, en effet, joue aujourd'hui le rôle qui était dévolu anciennement aux religions, à savoir de relier les individus entre eux au moyen d'un ralliement à des idées communément partagées. C'est ce qui permet à Durkheim de dire qu' "entre la science et la religion, il n'y a... que des différences de degrés ${ }^{18}$. En tant que représentation collective et institution sociale, la science est la mythologie des temps modernes. "Sans la civilisation, l'homme ne serait qu'un animal. C'est par la coopération et par la tradition sociales que l'homme s'est fait homme. Moralités, langages, religions, sciences sont des œeuvres collectives, des choses sociales. Or c'est par la moralité que l'homme forme en lui la volonté, qui dépasse le désir ; c'est le langage qui l'élève au-dessus de la pure sensation, c'est dans la religion d'abord, puis dans les sciences, que s'élaborent les notions cardinales dont est faite l'intelligence proprement humaine ${ }^{19}$. »

Le positiviste Durkheim s'empresse donc de conclure que la science ne représente pas une nécessité impérieuse pour l'homme. Celui-ci peut très bien s'en passer s'il dispose d'un discours cohérent autre et de " traditions fortes et respectées ${ }^{20}$ à même de structurer le tout social. C'est ainsi que le christianisme a pu naturellement magnifier les pauvres. C'est ainsi qu'aujourd'hui encore, une théorie scientifique n'est recevable et crédible que si lui préexiste un fort sentiment fait d'assentiment. Sans l'existence d'un courant d'opinion qui lui soit favorable, une démonstration scientifique a peu de chances de pouvoir convaincre. C'est sans réserves que l'" on peut s'accorder sur le fait que la raison a bien peu de place

17. Ibid.

18. É. Durkheim, Les formes élémentaires de la vie religieuse, PUF, Paris, 1979, p. 17.

19. P. Fauconnet, "Lœeuvre pédagogique de Durkheim. Introduction », in É. Durkheim, Éducation et sociologie (1922), PUF, Paris, 1985, p. 13.

20. É. Durkheim, Éducation et sociologie, PUF, Paris, 1985, p. 105. 
dans l'élaboration et la divulgation des opinions. La diffusion de celles-ci, que ce soit pour les premiers chrétiens ou pour les ouvriers socialistes du XIX ${ }^{e}$ siècle doit bien plus aux mécanismes de contagion du sentiment ou de l'émotion vécus en commun $^{21}$.»

Pas plus que l'histoire érigée en mode de connaissance positif, la science ne peut éliminer la part d'imaginaire dans la structuration de l'ordre social. "L'histoire s'efforce de rendre compte de la généalogie des événements en remontant la chaîne des causes qui les engendrent, mais les mythologies sont des généalogies de ces généalogies, elles tentent de remonter jusqu'aux ancêtres des temps euxmêmes. C'est pourquoi les Grecs confondirent vite Kronos, le père de Zeus, avec Cronos le Temps ${ }^{22}$. „ C'est donc pure illusion que de vouloir émanciper l'histoire au moyen d'une science qui la conçoit comme réalisation des « promesses du temps » en opposant un «logos libérateur à un mythos fabulateur ${ }^{23}$.

C'est ainsi qu'il faut comprendre que Platon recourt aux mythes sans forcément y croire, car il a peut-être eu l'intuition que ceux-ci racontaient l'histoire des hommes dans ce qu'elle a de pérenne, les rattachaient à une communauté de destin en leur parlant « du Grand Temps dans lequel nos durées retrouvent tous les seuils contre lesquels elles butent, que ce soit ceux de la naissance, de la mort, de l'amour, de la vérité, ou simplement celui de la présence de l'autre » ${ }^{24}$.

Aucun discours, quel qu'il soit, n'échappe à l'emprise du mythe : des discours de démystification du mythe, à l'instar du freudisme et du marxisme, ont pendant longtemps joué le rôle de mythes dans l'imaginaire des hommes. Avec la déréliction des mythes fondateurs de la modernité (le travail, la production, le bien-être matériel, le progrès, etc.) et des grands discours de référence, que Lyotard appelle les "métadiscours " ou les " métarécits " 25 (l'émancipation du citoyen chez les révolutionnaires français, la société sans classes chez Marx, l'augmentation de la richesse chez Smith, etc.), le savoir sociologique actuel n'est pas resté indifférent à la nouvelle sensibilité qui s'exprime dans notre postmodernité. La prégnance des mythes est telle qu'on pourrait concevoir l'histoire elle-même comme une succession ou une valse de mythes, comme l'éternel retour du même. En tout cas, c'est ainsi que l'a conçue toute une tradition philosophique, acquise à une réflexion se déployant hors du cadre étriqué des philosophies de l'histoire, qui va de Vico à Durand et Maffesoli en passant par Nietzsche.

Il nous semble difficile de ranger Durkheim parmi les positivistes purs et durs. Ne serait-ce qu'à cause, d'une part, de l'importance croissante que la religion revêt dans son ouvre sociologique, d'abord sous le vocable de conscience collective, ensuite sous celui de représentation collective, et, d'autre part, de la correspondance

21. M. Maffesoli, Le temps des tribus. Le déclin de l'individualisme dans les sociétés modernes, Méridiens Klincksieck, Paris, 1988, p. 26.

22. J. Brun, Philosophie de l'histoire. Les promesses du temps, Stock, Paris, 1990, p. 28.

23. Ibid., p. 29.

24. Ibid., p. 24.

25. J.-F. Lyotard, La condition postmoderne, Les Éditions de Minuit, Paris, 1985, p. 7. 
existant entre sa théorie de la connaissance et sa sociologie religieuse, correspondance qu'il établit lui-même dans la conclusion des Formes élémentaires, en ce sens que c'est bien la religion qui est à la source des formes de connaissance rationnelles, aussi bien de la philosophie que de la science.

La sociologie durkheimienne s'est fait forte de montrer que la société est une " machine à faire des dieux ${ }^{26}$. Cela veut dire qu'aucune société ne saurait perdurer et se reproduire sans que les hommes ne puissent se référer dans leur rapport à la nature et aux autres hommes à des facteurs imaginaires que Durkheim appelle idéaux, c'est-à-dire à l'existence d'un champ symbolique et affectif structurant leur être-ensemble. Cela veut dire aussi que les forces matérielles, à l'instar de l'économique, ne sont pas suffisantes pour souder l'être-ensemble : "Quand une institution, contraire à un principe de morale, ne se défend plus que par des raisons utilitaires, elle est condamnée et ne peut plus vivre longtemps ${ }^{27}$. "En de nombreuses occasions, Durkheim critique l'idée de l'économisme selon laquelle " la vie économique est apte à s'organiser d'elle-même, à fonctionner régulièrement et harmoniquement sans qu'aucune autorité morale lui soit proposée » 28 . Les représentations collectives sont les seules forces à être à même d'insuffler un dynamisme aux sociétés qui les empêche de dépérir sous le coup de l'entropie qui à plus longue échéance les menace toutes. Les croyances se retrouvent donc au centre de toutes les formes de socialisation, y compris dans les sociétés qui pensent avoir réussi à s'en passer, car elles représentent autant de points d'attache incontournables auxquels l'homme se réfère dans les situations d'interaction. Moscovici a remarquablement mis en lumière l'importance de ces croyances dans toute structuration sociale et souligné l'inanité de modèles, comme le paradigme moderne de l'homo oeconomicus, qui prétendent en faire l'économie, en présupposant des êtres agissant rationnellement. Il cite d'ailleurs un contemporain de Durkheim, Fustel de Coulanges, pour agrémenter sa démonstration : "Pour leur donner des règles communes [aux hommes], pour instituer le commandement et faire accepter l'obéissance, pour faire céder la passion à la raison et la raison individuelle à la raison publique, il faut assurément quelque chose de plus haut que la force matérielle et de plus respectable que l'intérêt, de plus sûr qu'une théorie philosophique, de plus immuable qu'une convention, quelque chose qui soit également au fond de tous les coeurs et qui y siège avec empire. Cette chose-là, c'est une croyance ${ }^{29}$. " Et d'ajouter le commentaire suivant : "Que cette croyance soit soutenue par un mythe, une idéologie ou une science, peu importe ; dès qu'elle existe, les hommes ressentent la vitalité du lien qui les unit, la force unique de leur conviction et l'aimant du but qui les fait agir ensemble ${ }^{30}$. »

26. Cf. S. Moscovici, La machine à faire des dieux, Fayard, Paris, 1988.

27. É. Durkheim, Le socialisme, PUF, Paris, 1971, p. 259.

28. É. Durkheim, Leçons de sociologie, PUF, Paris, 1990, p. 50.

29. Fustel de Coulanges, cité par S. Moscovici, La machine à faire des dieux, Fayard, Paris, 1988, p. 29.

30. Ibid. 
Si Durkheim accorde un tel crédit aux croyances, c'est bien parce qu'elles remplissent une fonction sociale qui rejette au second plan la question de leur véracité. Les raisons concrètes que les adeptes d'une religion, les partisans d'une politique, les défenseurs d'une éthique ou encore les serviteurs de la science invoquent sont peu de chose par rapport à la sincérité qui émane de leurs jugements. Prenant le contre-pied de Marx, pour qui « les hommes se sont toujours fait jusqu'ici des idées fausses sur eux-mêmes, sur ce qu'ils sont ou devraient être » et pour qui celles-ci ne sont que des " chimères, des idées, des dogmes, des êtres d'imagination qui les plient sous leur joug avilissant ${ }^{31}$, Durkheim montre que les croyances et les mythologies, qu'elles soient religieuses ou scientifiques, ne peuvent être tenues pour fausses, car toutes répondent à des besoins sociaux déterminés, toutes sont l'expression de la nature des choses : "C'est, en effet, un postulat essentiel de la sociologie qu'une institution humaine ne saurait reposer sur l'erreur et sur le mensonge : sans quoi elle n'aurait pas pu durer. Si elle n'était pas fondée dans la nature des choses, elle aurait rencontré dans les choses des résistances dont elle n'aurait pu triompher ${ }^{32}$.»

La grande étude de Durkheim sur les Formes élémentaires n'a d'ailleurs d'autre but que de mettre à nu la nature religieuse de l'homme et de mettre ainsi en relief les ressorts cachés de la vie humaine. La religion ne se caractérise donc pas de prime abord par l'existence d'un dieu ou de divinités personnelles, mais par un ensemble de croyances et de pratiques qui témoignent de l'existence du sacré en produisant des effets réels sur la vie des gens, effets qui sont quantifiables et mesurables. «Ce que nous trouvons à l'origine et à la base de la pensée religieuse, ce ne sont pas des objets ou des êtres déterminés et distincts qui possèdent par eux-mêmes un caractère sacré ; mais ce sont des pouvoirs indéfinis, des forces anonymes, plus ou moins nombreuses selon les sociétés, parfois même ramenées à l'unité, et dont l'impersonnalité est strictement comparable à celle des forces physiques dont les sciences de la nature étudient les effets. [...] Il n'est donc pas surprenant que, même dans les religions où il existe des divinités avérées, il y ait des rites qui possèdent une vertu efficace par eux-mêmes et indépendamment de toute intervention divine ${ }^{33}$. »

\section{Religion et société}

Si l'influence de la religion est mesurable en termes de forces, c'est bien parce qu'elle n'est autre chose que l'action salutaire que la société exerce sur l'individu. «Les tendances collectives ont une existence qui leur est propre; ce sont des forces aussi réelles que des forces cosmiques, bien qu'elles soient d'une autre nature ; elles agissent également sur l'individu du dehors, bien que ce soit par d'autres

31. K. Marx, Euvres, t. 3, Philosophie («L'idéologie allemande »), Gallimard, La Pléiade, Paris, 1982, p. 1049.

32. É. Durkheim, Les formes élémentaires de la vie religieuse, PUF, Paris, 1979, p. 3.

33. Ibid., pp. 285-286. 
voies. Ce qui permet d'affirmer que la réalité des premières n'est pas inférieure à celle des secondes, c'est qu'elle se prouve de la même manière, à savoir par la constance de leurs effets ${ }^{34}$. »

Cette force est purement symbolique et se manifeste sans même que l'on soupçonne sa présence. Le rapport de l'homme au monde est donc fondé sur quelque chose d'immatériel qui ne laisse pourtant pas d'être réel, sur ce que Michel Maffesoli nomme l' "imaginaire social ${ }^{35}$. Cet imaginaire nous permet de comprendre que ce qui relie les hommes entre eux n'est pas un dessein rationnel, de type contractuel ou conventionnel, mais un ensemble diffus de sentiments, de passions. Ces derniers se manifestent certes dans les moments paroxystiques, comme la Révolution française, dont même les plus dogmatiques des révolutionnaires ont dû reconnaître le caractère festif et sacré, comme le souligne Mona Ozouf, en ajoutant que ces transgressions festives ont « le mérite de faire apparaître l'emmêlement nécessaire, pour une sensibilité inchangée, de la cérémonie religieuse avec l'acte social » ${ }^{36}$, mais ils se manifestent aussi dans les moments plus discrets et anodins de la vie quotidienne, sous la forme du préjugé et du bon sens.

En termes durkheimiens, il s'agit donc de l'influence, tantôt massive, tantôt discrète, que la société exerce sur l'individu, que la conscience collective exerce sur la conscience individuelle, que les représentations exercent sur les institutions. La sociologie durkheimienne est née de l'idée que des recherches portant sur la société peuvent apporter un nouvel éclairage sur l'homme. Pour connaitre les sentiments et les croyances des hommes, il ne suffit pas d'en rester à l'analyse de la conscience individuelle, comme le fait la psychologie. Car l'homme n'est pas réductible aux facultés de sensation, d'émotion et de volition qu'il a en propre. Ne serait-ce que parce qu'il s'enrichit en permanence au moyen de leur exercice concret. Ce que nous trouvons en lui, ce sont des croyances religieuses, des règles juridiques, des maximes morales, des coutumes sociales qui, à l'instar de la technique, du langage et de la logique, le débordent de toutes parts. C'est parce que l'homme est porteur d'une expérience collective émanant d'un héritage culturel que la société transmet de génération en génération qu'il nous faut " penser le dynamisme humain à partir de ses racines, autre manière de dire la puissance de l'immémoriale tradition ${ }^{37}$. La transmission du patrimoine culturel de l'homme est donc le fait de la société, en tant qu'organisation permanente qui survit à la mort et à la succession des individus.

Aux deux caractéristiques de la société constatées dans les Règles, à savoir son extériorité et son aptitude à contraindre, Durkheim en évoque une troisième postérieurement, dans Les formes élémentaires, plus précisément sa capacité à

34. É. Durkheim, Le suicide. Étude de sociologie, PUF, Paris, 1973b, pp. 348-349.

35. M. Maffesoli, La contemplation du monde. Figures du style communautaire, Grasset, Paris, 1993, p. 157.

36. M. Ozouf, La fête révolutionnaire. 1789-1799, Gallimard, Paris, 1976, pp. 318-319.

37. M. Maffesoli, Le trésor caché. Lettre ouverte aux francs-maçons et à quelques autres, Éditions Léo Scheer, Paris, 2015, p. 88. 
produire des représentations. Cette idée, présente dans des écrits antérieurs, transparaissait auparavant sous la notion de conscience collective qui constitue une pièce maîtresse dans l'édifice conceptuel durkheimien. Ceux que cette notion aux contours diffus ne fait pas frémir ont par ailleurs souligné qu'elle est tout particulièrement appropriée pour dépeindre la socialité postmoderne : "La référence à Durkheim n'est pas inutile, sa notion de "conscience collective", si l'on n'en fait pas un concept intangible et une clef universelle, est tout à fait pertinente pour comprendre la société contemporaine et ses différentes effervescences, qui toutes s'effectuent autour ou à partir de sentiments, d'émotions, d'images, de symboles, causes et effets de cette "conscience collective" ${ }^{38}$. "

En reconnaissant la spécificité de la notion de conscience collective, Durkheim a mis sur pied une théorie de l'inconscient collectif dont la validité scientifique n'est plus mise en doute aujourd'hui. L'existence de l'inconscient collectif renvoie à l'idée que le psychisme collectif est sujet à des représentations au même titre que le psychisme individuel. "La vie collective, comme la vie mentale de l'individu est faite de représentations, il est donc présumable que représentations individuelles et représentations sociales sont, en quelque manière, comparables ${ }^{39}$. "Si tout est soumis à l'ordre de la représentation, on ne perçoit pas pour quelle raison objective la sphère de l'inconscient collectif s'y déroberait. La meilleure preuve que l'inconscient collectif n'est pas vaine chimère, c'est que dans la vie courante "nos jugements sont à chaque instant tronqués, dénaturés par des jugements inconscients ; nous voyons ce que nos préjugés nous permettent de voir et nous ignorons nos préjugés ${ }^{40}$. La notion d'inconscient collectif est donc le juste pendant de celle de conscience collective. En effet, c'est dans la mesure où la représentation collective s'impose aux hommes de l'extérieur qu'elle peut être dite inconsciente. Ni dans le domaine du psychisme individuel ni dans le domaine du psychisme collectif, les états mentaux ne sont réductibles à la conscience que nous en avons.

Contrariant les tenants d'une position purement matérialiste, Durkheim affirme que dans le monde social rien n'échappe à l'emprise de la représentation. «Dans la vie sociale, tout est représentations, tout est idées, sentiments, et nulle part on observe mieux la force efficace des représentations ${ }^{41}$. " Les faits sociaux sont à tel point constitués par des représentations, c'est-à-dire de valeurs, de croyances et de significations que même ceux qui relèvent de la morphologie sociale et qui renvoient à une pure matérialité, à l'exemple de la répartition des hommes dans l'espace ou de la division du travail, sont incorporés à elles. De la même façon que la conscience individuelle existe indépendamment de sa base, à savoir les sensations, en jouissant même d'une certaine autonomie, la conscience collective et les

38. M. Maffesoli, La contemplation du monde. Figures du style communautaire, Grasset, Paris, 1993, p. 148.

39. É. Durkheim, Sociologie et Philosophie, PUF, Paris, 1967, p. 2.

40. Ibid., p. 23.

41. É. Durkheim, Textes, tome 1, Éléments d'une théorie sociale, Les Éditions de Minuit, Paris, 1975, p. 61. 
représentations collectives qui en émanent se détachent progressivement de leur substrat, à savoir les consciences élémentaires, en leur permettant, par le jeu de l'action et de la rétroaction, de se combiner pour former un être nouveau, l'être social.

Dans le cas des représentations individuelles, comme dans celui des représentations collectives, la dépendance à l'égard d'un donné initial ne préjuge en rien de leur capacité à s'en affranchir. Cela est même plus évident pour les représentations collectives dans la mesure où l'extériorité des faits sociaux par rapport aux individus saute plus facilement aux yeux que celle des faits mentaux par rapport aux cellules cérébrales. Les faits sociaux sont en effet des manières de pensée, d'agir et de sentir, qui, tout en étant extérieurs aux consciences individuelles, s'imposent à elles. Lensemble des devoirs et des obligations auxquels nous nous assujettissons se trouvent consignés et codifiés dans les mours puis dans le droit, dans les coutumes puis dans la morale, avant même que nous les appliquions dans la pratique et alors même que nous nous félicitons d'agir exclusivement selon les desseins dictés par notre conscience.

C'est cette extériorité et cette antériorité des phénomènes sociaux par rapport aux individus qui leur confèrent un caractère d'obligation et qui permettent de comprendre "que ces manières d'agir et de penser ne sont pas l'œuvre de l'individu, mais émanent d'une puissance morale qui le dépasse, qu'on l'imagine mystiquement sous la forme d'un Dieu ou qu'on s'en fasse une conception plus temporelle et plus scientifique ${ }^{42}$. Le naturalisme sociologique de Durkheim n'est donc pas d'essence matérialiste, mais spiritualiste : "Si l'on appelle spiritualité la propriété distinctive de la vie représentative chez l'individu, on devra dire de la vie sociale qu'elle se définit par une hyperspiritualité ; nous entendons par là que les attributs constitutifs de la vie psychique s'y retrouvent, mais élevés à une bien plus haute puissance et de manière à constituer quelque chose d'entièrement nouveau $^{43}$. » La définition durkheimienne du fait social comme chose est donc loin de prendre une tonalité exclusivement positiviste, puisqu'elle s'ouvre sur une véritable psychologie sociale. La réalité à laquelle la notion de conscience collective renvoie est bien l'écho du groupe dans les consciences, l'immanence du tout aux parties. Mais le tout n'a nullement besoin d'être hypostasié en dehors des parties - même si les attributs d'extériorité et de contrainte qu'il possède pourraient nous y faire penser - pour que nous soyons à même de reconnaître la spécificité du social. En effet, le support du social, si on le comprend comme conscience collective, n'est jamais que l'ensemble des consciences associées.

Le fait que Durkheim substitue les termes de représentation ou d'opinion publique à celui de conscience collective ne change rien à la manière dont il les conçoit et les définit : "Lensemble des croyances et des sentiments communs à la moyenne des membres d'une même société forme un système déterminé qui a sa vie propre ; on peut l'appeler la conscience collective ou commune ${ }^{44}$. »

42. É. Durkheim, Sociologie et Philosophie, PUF, Paris, 1967, pp. 27-28.

43. Ibid., p. 37.

44. É. Durkheim, De la division du travail social, PUF, Paris, 1978, p. 46. 
Dans son approche des phénomènes sociaux, la sociologie ne peut donc rester de marbre à l'égard de ce qu'il est donné à l'homme de voir : toutes sortes d'institutions sociales, que ce soient des législations, des cultes ou encore des rites. Mais la sociologie ne peut s'arrêter à leur simple étude, elle doit pouvoir expliquer ce qui leur donne consistance et vie. C'est parce que «les croyances sont le noud vital de toute société » que la sociologie s'attellera à mettre en évidence les « états psychiques » plutôt que les «formes matérielles » par lesquelles ceux-là s'expriment, et qu'elle pourra être définie comme " une étude de l'opinion » ${ }^{45}$.

\section{Science et représentations collectives}

C'est aussi du point de vue d'une étude de l'opinion que la sociologie envisagera la science et son moyen de prédilection, le concept, dont le but est de sceller "l'accord des esprits les uns avec les autres, mais aussi, et plus encore, leur accord avec la nature des choses $"{ }^{46}$. Les immenses progrès accomplis par la science ne changent pas fondamentalement la donne, à savoir d'une part que la vérité est redevable à ses conditions sociales de production, et de ce point de vue Durkheim retrouve Marx, et d'autre part qu'elle détermine la consistance morale de l'êtreensemble dans la mesure où elle exprime le collectif dans l'individu. Le concept a une validité tangible non par les vérités intrinsèques qu'il peut exprimer, mais par l'autorité extrinsèque qu'il exerce sur tous ceux qui intègrent une communauté donnée. Aussi la valeur objective des concepts n'est-elle pas tant liée aux règles scientifiques qui président à leur élaboration qu'à l'autorité qu'elle tient de son accord harmonieux avec les autres croyances, en faisant bloc avec l'ensemble des autres représentations collectives. Le fait que le consensus social ne provienne plus de vérités apodictiques transmises par le mythe, mais de vérités scientifiques démontrées, le fait comme dirait Comte que même la religion se fasse positive, dans la mesure où on lui demande d'être démontrée, ne retire rien au caractère mythique de la science : "Le concept qui, primitivement, est tenu pour vrai parce qu'il est collectif tend à ne devenir collectif qu'à condition d'être tenu pour vrai : nous lui demandons ses titres avant de lui accorder nos créances ${ }^{47}$. »

La rupture avec le sens commun, que d'aucuns ont élevé au rang de pétition de principe servant d'unique garant à la scientificité de la science n'est pas de mise pour Durkheim. Ce qui permet aux concepts d'apparaître, de se généraliser et d'être tenus pour valides, c'est qu'ils sont des représentations collectives qui, en tant que collectives, sont garantes d'objectivité. Limportance dévolue au rôle de la science à notre époque est donc tributaire de la foi que nous plaçons en elle et de notre capacité à la concevoir à la manière d'un mythe fondateur de sociétés. Une théorie créée dans le but de connaître pour connaitre serait une ineptie, car

45. C. Bouglé, « Préface » à É. Durkheim, Sociologie et Philosophie, PUF, Paris, 1967, p. VII.

46. É. Durkheim, Les formes élémentaires de la vie religieuse, PUF, Paris, 1979, p. 624.

47. Ibid. 
les idées comme les choses ne sont jamais indépendantes les unes des autres. En somme, la connaissance pour la connaissance est aussi stérile que l'art pour l'art, car renvoyant à eux-mêmes ils se privent du rapport à la transcendance, fût-elle immanente, qui est le ciment de toute civilisation.

La citation suivante de Nietzsche, qui aurait pu être celle de Durkheim, revêt une signification tout à fait différente chez les deux auteurs : "Il est nécessaire que quelque chose soit tenu pour vrai, - mais il n'est nullement nécessaire que cela soit vrai $^{48}$. " Le premier est conduit à considérer les doctrines religieuses et leurs émanations sécularisées comme la démocratie, le socialisme et le libéralisme comme des pensées fausses, des formules faciles pour dénicher le bonheur au moyen d'un idéal de soumission qui n'est qu'un prétexte pour acquérir la puissance. En fait, ce que Nietzsche nomme de façon méprisante les "mondes illusoires " et les "êtres figurés ", ou ce que Durkheim qualifie de manière laudative de monde de l'idéal, sont les expressions de l'immatérialité constitutive de toute vie sociale, ce par quoi une société ou une civilisation tiennent. Il est donc normal que la conception durkheimienne d'une objectivité intrinsèque de la nature des choses, que la connaissance se contente de reproduire, engage aussi sa conception de la morale, en tant qu'hypostase des vertus collectives.

En fait, il faut bien reconnaitre que les groupes sociaux ont de tout temps construit des explications quant à leur présence dans le monde, leur rapport au monde et leurs rapports aux autres à l'intérieur de ce monde, explications qui fonctionnent comme autant d'ensembles significatifs à partir desquels ils organisent leurs interactions. Ces ensembles significatifs peuvent consister aussi bien en récits mythiques qu'en discours scientifiques. Un esprit analytique aura tendance à les départager en fonction des contenus qu'ils véhiculent, alors qu'une pensée synthétique, qu'un positiviste comme Comte appelait de ses vœux, restera davantage attentive à la " charge idéologique " ${ }^{49}$ qu'ils renferment et à leur capacité d'agréger les individus. Dans cette optique, il faut bien convenir du caractère profondément synergique, communautaire et même mythique, de la science : "C'est sa dimension mythique qui rend une idée dynamique, qui lui permet d'exalter les enthousiasmes et qui engendre des projets et des réalisations. En ce sens, c'est en tant que mythe que le scientisme du $\mathrm{XX}^{e}$ siècle a pu promouvoir les réalisations économico-technologiques que l'on sait ${ }^{50}$. "

La science déconsidère le mythe et la religion, mais elle n'est pas en mesure de se substituer à eux. Le fait qu'elle s'imagine que l'homme puisse se contenter des résultats objectifs qu'elle atteint et des applications pratiques qui en découlent n'est que pure illusion. S'il est loisible d'admettre qu'il n'appartient pas à la science de répondre aux questions existentielles que l'homme se pose de toute éternité, il

48. F. Nietzsche, La volonté de puissance. Essai d'une transmutation de toutes les valeurs, tome 2, Mercure de France, Paris, 1930, p. 18.

49. M. Maffesoli, "Présentation ", in É. Durkheim, Les formes élémentaires de la vie religieuse, Le Livre de Poche, Paris, 1991, p. 33.

50. Ibid. 
serait fallacieux de penser que l'homme cessera un jour de s'interroger sur le sens de son existence et de sa destinée. En d'autres termes, même un esprit des plus acquis à la science ne peut que constater la pérennité des mythes et des croyances. C'est à juste titre que Gilbert Durand remarque que le positivisme de Durkheim ne l'a nullement empêché de pressentir « l'importance générique du récit sacré, de la religion, comme indice majeur d'une société : les dieux et les mythes expriment la prégnance sociale de tout lien sociétal ${ }^{51}$.

Ce que l'on a dit du discours scientifique vaut également pour le message religieux. Ce n'est pas tant le contenu des dogmes religieux que la participation communielle, magique et cosmique au tout social, qui importe. C'est donc à la faveur des mythes et des rites, c'est-à-dire de la propagation émotionnelle et affective de la foi, que les religions exercent leur influence sociale. Lors des moments d'effervescence religieuse, c'est bien la société qui transcende l'individu, la conscience collective le marquant de son empreinte, le sacré prenant possession de lui, les vertus collectives se propageant en lui. Les forces religieuses « ne sont, en effet, que des forces collectives hypostasiées, c'est-à-dire des forces morales; elles sont faites des idées et des sentiments qu'éveille en nous le spectacle de la société, non des sensations qui nous viennent du monde physique ${ }^{52}$. Faut-il rappeler que les statistiques que Durkheim utilise à profusion dans Le suicide ne servent d'ailleurs qu'à attester de la manière dont la conscience collective imprègne les consciences individuelles : le mariage, le divorce, la procréation, le crime, le suicide n'existent pas à l'état isolé, ils ne sont nullement redevables à des " inclinations personnelles ${ }^{53}$, mais obéissent en fait à des tendances sociétales de fond qui dépassent les individus et les entraînent dans un même mouvement sans même qu'ils en aient conscience.

Lun des postulats majeurs de la sociologie durkheimienne consiste donc dans l'idée de l'irréductibilité de la conscience collective à la conscience individuelle. En effet, les croyances et les sentiments sont redevables à une réalité plus vaste que celle de la conscience individuelle et si cette dernière y participe elle ne la crée pas. Si l'homme ne se suicide pas en masse, c'est parce que l'autorité de la conscience collective lui fait sentir que la vie vaut mieux que la mort. S'il ne s'adonne pas au crime, c'est que la conscience collective imprime en lui le sens du devoir moral. À l'inverse, s'il existe des formes de transgressions socialement reconnues, qui font que "l'homme devient autre » ${ }^{54}$, alors qu'elles sont répréhensibles du point de vue de la routine et des critères normatifs, c'est parce que la vie collective y gagne une nouvelle dimension et qu'elle se régénère socialement par la prise de conscience des vertus agrégatives du groupe. À ce titre, les croisades, les révolutions, les carnavals sont autant de moments d'effervescence qui fonctionnent comme des catalyseurs de l'énergie vitale des individus et donnent une nouvelle consistance à la

51. G. Durand, Champs de l'imaginaire, Ellug, Grenoble, 1996, p. 113.

52. É. Durkheim, Les formes élémentaires de la vie religieuse, PUF, Paris, 1979, p. 461.

53. É. Durkheim, L'Éducation morale, Fabert, Paris, 2005, p. 287.

54. É. Durkheim, Les formes élémentaires de la vie religieuse, PUF, Paris, 1979, p. 301. 
fusion de l'être-ensemble. Ils ne représentent au fond que la marque de l'idéalité s'élaborant dans la conscience collective et s'inscrivant dans le réel, c'est-à-dire dans les choses, le temps, les lieux, le langage, etc. Ils ne sont que le prétexte d'une renaissance : "Une Renaissance, c'est de la vie sociale qui, après s'être comme déposée dans des choses et y être restée longtemps latente, se réveille tout à coup et vient changer l'orientation intellectuelle et morale de peuples qui n'avaient pas concouru à l'élaborer. Sans doute, elle ne pourrait pas se ranimer si des consciences vivantes ne se trouvaient là pour recevoir son action ; mais, d'un autre côté, ces consciences auraient pensé et senti tout autrement si cette action ne s'était pas produite ${ }^{55}$. „C'est bien de cela dont il est question dans l'explication que Durkheim donne du social en acte : l'imaginaire est au fondement même de toute forme de reliance.

\section{Bibliographie}

Besnard P., Lanomie, ses usages et ses fonctions dans la discipline sociologique depuis Durkheim, PUF, Paris, 1987.

Bouglé C., "Préface », in É. Durkheim, Sociologie et Philosophie, PUF, Paris, 1967, pp. V-XI.

Brun J., Philosophie de l'histoire. Les promesses du temps, Stock, Paris, 1990.

Durand G., Champs de l'imaginaire, Ellug, Grenoble, 1996.

Durkheim É., Montesquieu et Rousseau précurseurs de la sociologie, Marcel Rivière, Paris, 1966.

Durkheim É., Sociologie et Philosophie, PUF, Paris, 1967.

Durkheim É., Journal sociologique, PUF, Paris, 1969.

Durkheim É., La science sociale et l'action, PUF, Paris, 1970.

Durkheim É., Le socialisme, PUF, Paris, 1971.

Durkheim É., Les règles de la méthode sociologique, PUF, Paris, 1973a.

Durkheim É., Le suicide. Étude de sociologie, PUF, Paris, 1973b.

Durkheim É., Textes, tome 1, Éléments d'une théorie sociale, Les Éditions de Minuit, Paris, 1975.

Durkheim É., De la division du travail social, PUF, Paris, 1978.

Durkheim É., Les formes élémentaires de la vie religieuse, PUF, Paris, 1979.

Durkheim É., Éducation et sociologie, PUF, Paris, 1985.

Durkheim É., Leçons de sociologie, PUF, Paris, 1990.

Durkheim É., L'Éducation morale, Éditions Fabert, Paris, 2005.

Fauconnet P., «L Lœuvre pédagogique de Durkheim. Introduction », in É. Durkheim, Éducation et sociologie (1922), PUF, Paris, 1985, pp. 11-40.

Freund J., Philosophie et sociologie, Cabay, Louvain-la-Neuve, 1984.

Lacroix B., Durkheim et le politique, Presses de la fondation nationale des sciences politiques (Université de Montréal), Montréal, 1981.

Lyotard J.-F., La condition postmoderne, Les Éditions de Minuit, Paris, 1985.

55. É. Durkheim, Le suicide. Étude de sociologie, PUF, Paris, 1973b, pp. 354-355. 
Maffesoli M., Le temps des tribus. Le déclin de l'individualisme dans les sociétés modernes, Méridiens Klincksieck, Paris, 1988.

Maffesoli M., « Présentation » de Durkheim É., Les formes élémentaires de la vie religieuse, Le Livre de Poche, Paris, 1991, pp. 5-36.

Maffesoli M., La contemplation du monde. Figures du style communautaire, Grasset, Paris, 1993.

Maffesoli M., Le trésor caché. Lettre ouverte aux francs-maçons et à quelques autres, Éditions Léo Scheer, Paris, 2015.

Marx K., Euvres, tome 3, Philosophie, Gallimard, La Pléiade, Paris, 1982.

Moscovici S., La machine à faire des dieux, Fayard, Paris, 1988.

Nietzsche F., La volonté de puissance. Essai d'une transmutation de toutes les valeurs, tome 2, Mercure de France, Paris, 1930.

Ozouf M., La fête révolutionnaire. 1789-1799, Gallimard, Paris, 1976. 